\title{
CINÉTICA DE ABSORÇÃO DE AMÔNIO E CRESCIMENTO RADICULAR DAS CULTIVARES DE ARROZ AGULHA E BICO GANGA ${ }^{1}$
}

\author{
JANE DEARAUJO BAPTISTA ${ }^{2}$, MANLIO SILVESTRE FERNANDES ${ }^{3}$ e SONIA REGINA DE SOUZA ${ }^{4}$
}

\begin{abstract}
RESUMO - Este trabalho teve por objetivo avaliar, em dois estádios de desenvolvimento, o crescimento do sistema radicular e a cinética de absorção de amônio das cultivares de arroz Agulha e Bico Ganga, procedentes do Estado do Maranhão. As plantas ( 25 e 50 dias de idade) cresceram em casa de vegetação, em solução nutritiva, com quatro níveis de $\mathrm{N}\left(20,40,60\right.$ e $\left.80 \mathrm{mg} \mathrm{L}^{-1}\right)$. Os valores de Vmáx, $\mathrm{K}_{\mathrm{M}}$ e Cmín foram determinados empregando-se um processo gráfico-matemático. A cultivar Bico Ganga apresentou maiores valores de Vmáx e menores de Cmín, aos 25 e 50 dias de idade, nos níveis de 20 e $40 \mathrm{mg} \mathrm{L}^{-1}$ de $\mathrm{N}$, enquanto em altos níveis de $\mathrm{N}\left(60\right.$ e $\left.80 \mathrm{mg} \mathrm{L}^{-1}\right)$, os maiores valores de Vmáx e menores de Cmín ocorreram na cultivar Agulha. Tanto aos 25 quanto aos 50 dias, a Bico Ganga apresentou maior área radicular, maior produção de matéria fresca e seca de raiz e parte aérea, e maior efíciência de acumulação de $\mathrm{N}$ e síntese de proteína na parte aérea. Esses resultados sugerem que a Bico Ganga, por ser uma cultivar local mais antiga que a Agulha, estaria melhor adaptada ao estresse nutricional em ambientes tropicais.
\end{abstract}

Termos para indexação: Oryza sativa, sistema radicular, solução nutritiva, absorção de nutrientes, nitrogênio.

\author{
AMMONIUM UPTAKE KINETICS AND ROOT GROWTH \\ OF THE RICE CULTIVARS AGULHA AND BICO GANGA
}

\begin{abstract}
A study was made on the patterns of root growth and the kinetics of N-uptake of the cultivars of tropical rice Agulha and Bico Ganga, from the Northeastern state of Maranhão, Brazil. Plants ( 25 and 50 days old) were grown in a greenhouse in nutrient solution. Four levels of $\mathrm{N}$ were used $\left(20,40,60\right.$ and $\left.80 \mathrm{mg} \mathrm{L}^{-1}\right)$. Vmax, $\mathrm{K}_{\mathrm{M}}$ and $\mathrm{Cmin}$ were determined by a mathematical graphic process. Bico Ganga had the highest Vmax and the lowest Cmin, both at 25 and 50 days at 20 and $40 \mathrm{mg} \mathrm{L}^{-1}$ of N. However, at higher levels of $\mathrm{N}$ ( 60 and $\left.80 \mathrm{mg} \mathrm{L}^{-1}\right)$ the inverse was observed, Agulha had higher Vmax and lower Cmin. At 25 and 50 days Bico Ganga showed higher total root area and higher root dry and fresh weight. Also, Bico Ganga was more efficient in $\mathrm{N}$ accumulation and protein synthesis. As Bico Ganga is an older local variety than Agulha, it seems that those characteristics indicate a better adaptation of the former to the nutrient stress of the tropical environment were it has been grown.
\end{abstract}

Index terms: Oryza sativa, root systems, nutrient solutions, nutrient uptake, nitrogen.

${ }^{1}$ Aceito para publicação em 7 de dezembro de 1999.

${ }^{2}$ Eng. Agrôn., M.Sc., Dep. de Solos, Universidade Federal Rural do Rio de Janeiro (UFRRJ), CEP 23890-000 Seropédica, Rio de Janeiro, RJ. E-mail: baptista@ufrrj.br

${ }^{3}$ Eng. Agrôn., Ph.D., Dep. de Solos, UFRRJ

E-mail: manlio@ufrrj.br

${ }^{4}$ Eng. Agrôn., Ph.D., Dep. de Química, UFRRJ.

E-mail: soniabq@ufrrj.br

\section{INTRODUÇÃO}

Em muitas espécies de plantas, a variação dos parâmetros cinéticos de absorção $\mathrm{K}_{\mathrm{M}}$ (constante de Michaelis-Menten) Vmáx (velocidade máxima) e Cmín (concentração mínima) é ampla e está relacionada a morfologia, fisiologia e desenvolvimento do sistema radicular, o que significa indicativo de diferenças 
genotípicas quanto à capacidade de adaptação aos diversos ecossistemas.

Entre os fatores que afetam a absorção de nutrientes pelas plantas, parâmetros morfológicos do sistema radicular têm sido apontados como importantes na caracterização de espécies e cultivares quanto à eficiência de absorção de nutrientes, destacando-se a área e o comprimento das raízes (Tachibana \& Ohta, 1983; Vilela \& Anghinoni, 1984; Anghinoni et al., 1989; Hoffand et al., 1990).

Alterações na morfologia e fisiologia radicular são comuns em plantas cultivadas. Anghinoni et al. (1989), estudando parâmetros morfológicos de raiz e de absorção dos nutrientes $\mathrm{P}, \mathrm{K}, \mathrm{Ca}$ e Mg em cultivares de milho, arroz e trigo, verificaram variação dos parâmetros cinéticos, sendo Vmáx o de maior variabilidade. As diferenças observadas foram atribuídas à menor ou maior capacidade de desenvolvimento do sistema radicular de cada espécie ou genótipo.

Como a velocidade de absorção de nutrientes é função do número de carreadores e de sua velocidade de giro, o aumento do sistema radicular resulta em maior número de sítios de absorção, além de proporcionar exploração de maior volume de solo, com conseqüentes alterações nos demais parâmetros cinéticos de absorção (Fontes \& Barber, 1984; Clarkson, 1985). Steingrobe \& Schenk (1993), estudando a demanda de $\mathrm{N}$ por plantas de alface, em função do estádio de desenvolvimento, observaram correlação positiva entre o incremento do influxo máximo de $\mathrm{N}$ (Vmáx) e o aumento do peso de matéria seca das raízes.

Avaliando duas cultivares de batata, Sattelmacher et al. (1990) observaram maior taxa de absorção de N por unidade de área radicular na cultivar Bodenkraft, considerada ineficiente quanto ao uso de $\mathrm{N}$, e menor taxa de absorção de $\mathrm{N}$ na cultivar Astrid, considerada eficiente quanto ao uso de $\mathrm{N}$, a qual apresentou sistema radicular amplo. Esse fato levou à suposição de que a maior superfície radicular possibilitou à cultivar Astrid explorar maior área de solo, e, conseqüentemente, aumentar a eficiência na absorção e utilização de $\mathrm{N}$.

O efeito da morfologia do sistema radicular sobre a absorção de $\mathrm{N}$ depende da superfície de absorção do sistema radicular, do volume de solo explorado e da concentração de $\mathrm{N}$ na solução do solo. Em solos minerais, com baixa concentração de $\mathrm{N}$, comuns nas regiões tropicais, a densidade radicular pode desempenhar papel relevante no processo de absorção de N (Schenk \& Barber, 1979).

Este trabalho teve por objetivo avaliar, em dois estádios de desenvolvimento, o crescimento do sistema radicular e a cinética de absorção de amônio das cultivares de arroz Agulha e Bico Ganga.

\section{MATERIAL E MÉTODOS}

O experimento foi conduzido em casa de vegetação, no Departamento de Solos da Universidade Federal Rural do Rio de Janeiro, Seropédica, RJ. Utilizou-se delineamento experimental inteiramente casualizado, com seis repetições, sendo os tratamentos constituídos pelas combinações de duas cultivares de arroz e de quatro níveis de $\mathrm{N}$ $\left(20,40,60\right.$ e $\left.80 \mathrm{mg} \mathrm{L}^{-1}\right)$

Foram utilizadas as cultivares de arroz Agulha e Bico Ganga, procedentes do Estado do Maranhão, ambas medianamente tolerantes ao alumínio (Mendonça, 1991) e com ciclo de 120 dias, apresentando 6,38\% e $11,21 \%$ de proteína bruta (Ferraz Júnior, 1993), e produtividade de 1.500 e $1.800 \mathrm{~kg} \mathrm{ha}^{-1}$, respectivamente

As sementes de arroz foram colocadas para germinar em uma mistura de areia e vermiculita, na proporção de $2: 1$. Cinco dias após a germinação, quatro plântulas selecionadas quanto à uniformidade foram transplantadas para vasos com capacidade para $4 \mathrm{~L}$ contendo solução nutritiva ( $\mathrm{pH} 6,0 \pm 0,1$, a um quarto da concentração original) (Hoagland \&Arnon, 1950). Após cinco dias, substituiu-se essa solução por outra com metade da concentração original. Tal procedimento foi utilizado para evitar estresse às radículas de arroz, bastante sensíveis a choques salino (Furlani \& Furlani, 1988).

Decorridos quatro dias, as plantas foram novamente selecionadas e transferidas para recipientes contendo $2 \mathrm{~L}$ de solução nutritiva na concentração original (Hoagland \& Arnon, 1950). A renovação da solução foi feita a cada três dias e o pH corrigido diariamente. Setenta e duas horas antes do início do estudo da cinética de absorção de amônio, plantas inteiras de arroz, com 25 ou 50 dias, foram transferidas para vasos de plástico com $0,5 \mathrm{~L}$ de solução desprovida de $\mathrm{N}$, para aumentar a capacidade de as raízes absorver o elemento (Lee \& Rudge, 1986). Em seguida, determinaram-se os parâmetros cinéticos Vmáx $\mathrm{K}_{\mathrm{M}} \mathrm{e}$ Cmín, empregando-se o processo gráfico-matemático proposto por Ruiz (1985), baseado no método de Claassen \& Barber (1974). 
Durante um período de sete horas, foram feitas coletas a cada 30 minutos, retirando-se alíquotas de $5 \mathrm{~mL}$ de solução com a finalidade de obter as curvas de depleção do íon amônio no período. Decorridas 24 horas do início das coletas, realizou-se a última amostragem das soluções nutritivas para obtenção de Cmín. Os valores de Vmáx e $\mathrm{K}_{\mathrm{M}}$ foram calculados utilizando-se o programa Cinética (Ruiz $\&$ Fernandes Filho, 1992). Depois de definido, o modelo de melhor ajuste foi utilizado para todas as concentrações

As concentrações de amônio em solução foram determinadas por destilação sob arraste de vapor, e posteriormente tituladas (Jones Junior, 1991).

Após as amostragens das soluções, as plantas foram colhidas e separadas em parte aérea e raízes, determinando-se os pesos de matéria fresca e seca. A área radicular foi determinada pelo método fotoelétrico (Rossiello et al., 1995).

\section{RESULTADOS E DISCUSSÃO}

A cultivar Bico Ganga apresentou, aos 25 dias de idade, maior área radicular, com raízes longas e finas, enquanto Agulha apresentou sistema radicular menor, constituído por raízes mais grossas e curtas. Na avaliação de plantas com 50 dias, a cultivar Bico Ganga apresentou raízes mais grossas e maior área radicular do que a cultivar Agulha (Tabela 1). $\mathrm{O}$ maior desenvolvimento do sistema radicular apresentado pela cultivar Bico Ganga é uma característica desejável, principalmente para cultivo sob condições de baixa fertilidade de solo. Essa adaptação morfológica do sistema radicular pode ser uma es- tratégia para aumentar a eficiência de absorção de nutrientes, considerando-se o maior tamanho do sistema radicular um indicativo de uma maior taxa de penetração de raízes no solo, o que garantiu maior absorção de nutrientes presentes em horizontes mais afastados da superfície do solo (Clarkson, 1985).

A cultivar Bico Ganga apresentou maiores produções de matéria fresca e seca das raízes, tanto aos 25 como aos 50 dias (Tabela 1). Segundo Mengel \& Barber (1974), a capacidade diferenciada entre cultivares em desenvolver o sistema radicular pode ser interpretada como uma habilidade adaptativa para absorver nutrientes mais eficientemente.

$\mathrm{Na}$ Tabela 1 observa-se, ainda, que a cultivar Bico Ganga apresentou menor relação raiz:parte aérea que a cultivar Agulha aos 25 dias, enquanto nas plantas com 50 dias o comportamento foi inverso. Em relação à idade das plantas, houve diminuição da relação raiz:parte aérea em ambas as cultivares, sendo a magnitude do decréscimo menor em relação à Bico Ganga, mostrando que as plantas dessa cultivar deslocaram maiores quantidades de matéria seca e fresca para as raízes.

O aumento de matéria seca das raízes e a redução relativa do peso de matéria seca da parte aérea apresentados pela cultivar Bico Ganga, quando comparada à Agulha, podem ser interpretados como uma adaptação da planta a ambientes com escassez de N, obrigando-a a manter tecidos radiculares viáveis na tentativa de viabilizar o influxo de água, $\mathrm{N}$ e outros nutrientes necessários ao crescimento. A capacida-

TABELA 1. Produção de matéria fresca e seca das raízes e da parte aérea e relação raiz:parte aérea de cultivares de arroz, com 25 e 50 dias 1.

\begin{tabular}{|c|c|c|c|c|c|c|}
\hline \multirow[t]{2}{*}{ Cultivar } & \multirow[t]{2}{*}{$\begin{array}{l}\text { Área radicular } \\
\qquad\left(\mathrm{cm}^{2}\right)\end{array}$} & \multicolumn{2}{|c|}{$\begin{array}{l}\text { Matéria fresca } \\
\left(\mathrm{g} \mathrm{planta}^{-1}\right)\end{array}$} & \multicolumn{2}{|c|}{$\begin{array}{c}\text { Matéria seca } \\
\left(\mathrm{g} \text { planta }^{-1}\right)\end{array}$} & \multirow[t]{2}{*}{$\begin{array}{c}\text { Relação } \\
\text { raiz: parte aérea }\end{array}$} \\
\hline & & Raiz & Parte aérea & Raiz & Parte aérea & \\
\hline & \multicolumn{6}{|c|}{25 dias } \\
\hline Agulha & $69,73 b$ & $0,51 b$ & $0,59 b$ & $0,04 \mathrm{ab}$ & $0,38 \mathrm{~b}$ & $0,86 a$ \\
\hline Bico Ganga & $92,45 \mathrm{a}$ & $0,59 \mathrm{a}$ & $0,75 \mathrm{a}$ & $0,06 \mathrm{a}$ & $0,61 \mathrm{a}$ & $0,78 \mathrm{~b}$ \\
\hline C.V.(\%) & 12,78 & 9,68 & 6,20 & 9,30 & 11,36 & 10,36 \\
\hline & \multicolumn{6}{|c|}{50 dias } \\
\hline Agulha & $161,60 \mathrm{a}$ & 2,25 & $5,97 \mathrm{~b}$ & $0,22 b$ & $0,89 b$ & $0,37 \mathrm{~b}$ \\
\hline Bico Ganga & $263,88 b$ & $3,54 \mathrm{a}$ & $7,65 \mathrm{a}$ & $0,44 \mathrm{a}$ & $1,22 \mathrm{a}$ & $0,46 \mathrm{a}$ \\
\hline C.V.(\%) & 6,45 & 13,20 & 10,19 & 12,10 & 13,11 & 12,61 \\
\hline
\end{tabular}

${ }^{1}$ Médias seguidas de letras iguais na coluna não diferem significativamente pelo teste de Tukey a $5 \%$ de probabilidade. 
de genética de algumas plantas em desenvolver um sistema radicular com características adequadas ao ambiente em que são cultivadas representa, segundo Clark (1977), adaptação a condições de estresse, o que possibilita maior absorção de água e de sais minerais.

Observa-se na Tabela 2 que não houve diferenças nos teores de N-total das raízes entre as cultivares estudadas. Em relação aos teores N-total presentes na parte aérea, a cultivar Bico Ganga apresentou valores superiores aos da cultivar Agulha, tanto aos 25 quanto aos 50 dias da germinação. Praticamente não houve variação no teor de N-total nas duas cultivares com relação ao estádio de desenvolvimento.

A cultivar Bico Ganga apresentou maior teor de N-protéico que a cultivar Agulha, aos 25 e 50 dias (Tabela 1). De acordo com a literatura, o melhor nível de N para crescimento e produção da cultura do ar-

TABELA 2. Teores $\left(\mathrm{mg} \mathrm{Mg}^{-1}\right)$ de $\mathrm{N}$ na parte aérea e raízes e $\mathrm{N}$-protéico na parte aérea de cultivares de arroz em duas épocas, aos 25 e 50 dias após a germinação ${ }^{1}$.

\begin{tabular}{|c|c|c|c|c|c|c|}
\hline \multirow[t]{3}{*}{ Cultivar } & \multicolumn{4}{|c|}{$\mathrm{N}$-total } & \multirow{2}{*}{\multicolumn{2}{|c|}{$\begin{array}{l}\text { N-protéico } \\
\text { (parte aérea) }\end{array}$}} \\
\hline & \multicolumn{2}{|c|}{ Raiz } & \multicolumn{2}{|c|}{ Parte aérea } & & \\
\hline & 25 & 50 & 25 & 50 & 25 & 50 \\
\hline Agulha & $16,3 a$ & $19,6 \mathrm{a}$ & $28,9 b$ & $20,93 b$ & $29,4 \mathrm{~b}$ & $19,93 b$ \\
\hline Bico Ganga & $16,6 \mathrm{a}$ & $18,9 \mathrm{a}$ & $30,6 \mathrm{a}$ & $22,39 a$ & $32,5 \mathrm{a}$ & $23,51 \mathrm{a}$ \\
\hline C.V. (\%) & 6,68 & 4,39 & 6,62 & 7,35 & 7,46 & 6,33 \\
\hline
\end{tabular}

roz está em torno de 20 ppm de N (Fernandes, 1974, 1991), concordando com os resultados encontrados neste trabalho.

Barraclough (1993) estudando o conteúdo de nutrientes em plantas de centeio como indicador da eficiência nutricional, classificou as plantas que apresentaram níveis de $\mathrm{N}$ na forma de nitrato, na parte aérea, de 2, 9 e $144 \mathrm{mM}\left(6,3,28\right.$ e $455 \mathrm{mg} \mathrm{kg}^{-1}$ de $\mathrm{N}$ de matéria fresca) e níveis de nitrato, no sistema radicular, de 1,2 e $58 \mathrm{mM}\left(3,1,6,3\right.$ e $183 \mathrm{mg} \mathrm{kg}^{-1}$ de $\mathrm{N}$ de matéria fresca) como plantas deficientes, suficientes e abundantes em $\mathrm{N}$, respectivamente.

Os valores dos parâmetros cinéticos de absorção de $\mathrm{N}$ variaram de acordo com a concentração do nutriente na solução (Tabela 3). O valor de Vmáx obtido com $20 \mathrm{mg} \mathrm{L}^{-1}$ de $\mathrm{N}$ foi maior na cultivar Bico Ganga, aos 25 e 50 dias da germinação, havendo uma inversão de comportamento nos níveis mais elevados de $\mathrm{N}$ (60 e $80 \mathrm{mg} \mathrm{L}^{-1}$ ), quando a cultivar Agulha apresentou os maiores valores de Vmáx. Em relação ao estádio de desenvolvimento das plantas, aos 50 dias, os valores de Vmáx em relação à cultivar Agulha aumentaram em $25 \%$ e $147 \%$ (20 e $80 \mathrm{mg} \mathrm{L}^{-1} \mathrm{de} \mathrm{N}$ ), comparativamente às plantas com 25 dias. $\mathrm{Na}$ cultivar Bico Ganga, os aumentos foram de $88 \%$ e $152 \%$, respectivamente. Steingrobe \& Schenk (1993), estudando a demanda de $\mathrm{N}$ por plantas de alface, em função de seu estádio de desenvolvimento, observaram correlação positiva entre a Vmáx, que representa incremento do influxo máximo de $\mathrm{N}$, e o aumento do peso de matéria seca das raízes.

TABELA 3. Parâmetros Vmáx, $K_{M}$ e Cmín em plantas de arroz, cultivares Agulha e Bico Ganga, com 25 e 50 dias, submetidas a quatro níveis de $\mathrm{N}$ em solução nutritiva ${ }^{1}$.

\begin{tabular}{|c|c|c|c|c|c|c|}
\hline \multirow[t]{2}{*}{$\begin{array}{l}\text { Concentração N } \\
\left(\mathrm{mg} \mathrm{L}^{-1}\right)\end{array}$} & \multicolumn{2}{|c|}{$\begin{array}{c}\text { V máx } \\
\left(\mu \text { mol L L }^{-1} h^{-1}\right)\end{array}$} & \multicolumn{2}{|c|}{$\begin{array}{c}\mathrm{K}_{\mathrm{M},} \\
\left(\mathrm{mmol} \mathrm{L}{ }^{-1}\right)\end{array}$} & \multicolumn{2}{|c|}{$\begin{array}{c}\text { Cmín } \\
\left(\mathrm{mmol} \mathrm{L}^{-1}\right)\end{array}$} \\
\hline & Agulha & Bico Ganga & Agulha & Bico Ganga & Agulha & Bico Ganga \\
\hline & \multicolumn{6}{|c|}{25 dias } \\
\hline 20 & $16,27 b$ & $22,10 \mathrm{a}$ & $0,513 b$ & $0,577 \mathrm{a}$ & $0,252 \mathrm{a}$ & $0,222 b$ \\
\hline 40 & $28,50^{\mathrm{ns}}$ & $29,50^{\mathrm{ns}}$ & $1,061 \mathrm{a}$ & $0,867 b$ & $0,868 \mathrm{a}$ & $0,828 b$ \\
\hline 60 & $34,20 \mathrm{a}$ & $32,90 \mathrm{ab}$ & $2,796^{\mathrm{ns}}$ & $2,691^{\mathrm{ns}}$ & $1,377 \mathrm{~b}$ & $1,537 \mathrm{a}$ \\
\hline 80 & $54,60 \mathrm{a}$ & $44,29 \mathrm{~b}$ & $3,514 \mathrm{~b}$ & $4,510 \mathrm{a}$ & $2,049 \mathrm{~b}$ & $2,134 \mathrm{a}$ \\
\hline & \multicolumn{6}{|c|}{50 dias } \\
\hline 20 & $20,31 b$ & $41,70 \mathrm{a}$ & $0,836 \mathrm{a}$ & $0,518 b$ & $0,389 a$ & $0,119 b$ \\
\hline 40 & $32,40 \mathrm{~b}$ & $35,50 \mathrm{a}$ & $2,044 a$ & $1,645 b$ & $1,606 \mathrm{a}$ & $0,708 \mathrm{~b}$ \\
\hline 60 & $100,60 \mathrm{a}$ & $52,20 \mathrm{~b}$ & $3,450 \mathrm{a}$ & $2,938 b$ & $1,208 b$ & $1,374 \mathrm{a}$ \\
\hline 80 & $134,81 \mathrm{a}$ & $111,60 \mathrm{~b}$ & $3,517^{\mathrm{ns}}$ & $3,582^{\text {ns }}$ & $1,873 b$ & $2,880 \mathrm{a}$ \\
\hline
\end{tabular}

\footnotetext{
1 Médias seguidas de letras iguais na mesma linha, para cada parâmetro, não diferem significativamente pelo teste de Tukey a 5\% de probabilidade
} 
A cultivar Agulha apresentou, tanto aos 25 quanto aos 50 dias de idade, com 20 e $40 \mathrm{mg} \mathrm{L}^{-1}$ de $\mathrm{N}$, menores valores de Vmáx que a cultivar Bico Ganga. Segundo Anghinoni et al. (1989), valores mais baixos de Vmáx em uma mesma espécie são indicativos de menor eficiência de absorção em condições de alta concentração de nutrientes em solução. Assim, pode-se supor que, em condições de baixos teores de N, a cultivar de arroz Agulha é menos eficiente na absorção de $\mathrm{N}$ do que Bico Ganga.

Em ambas as épocas de coleta, a cultivar Bico Ganga apresentou valores de Cmín menores que os da Agulha, quando submetidas a níveis de $\mathrm{N}$ de 20 e $40 \mathrm{mg} \mathrm{L}^{-1}$. Como Cmín representa a concentração mínima a partir da qual a planta começa a absorver nutrientes da solução, quanto menor seu valor em uma dada cultivar, maior será sua capacidade de adaptação a ambientes com baixa disponibilidade de nutrientes. Desse modo, a cultivar Bico Ganga estaria melhor adaptada a condições de baixa disponibilidade de $\mathrm{N}$; quando submetida a concentrações mais elevadas de $\mathrm{N}$ a eficiência de absorção seria reduzida.

$O$ parâmetro $K_{M}$ aparente não apresentou tendência de inversão de valores com a elevação do nível de N como observado em Vmáx e Cmín. Os valores de $\mathrm{K}_{\mathrm{M}}$ variaram entre cultivares, níveis de $\mathrm{N}$ na solução e estádio de desenvolvimento das plantas. Entre os parâmetros cinéticos, tem-se observado que $\mathrm{K}_{\mathrm{M}} \mathrm{e}$ o que apresenta maior variação interespecífica (Anghinoni et al., 1989).

A combinação de alto valor para Vmáx ou baixo valor para $\mathrm{K}_{\mathrm{M}} \mathrm{e}$ Cmín, e maior área radicular são desejáveis nas cultivares, pois significa maior eficiência de absorção de nutrientes. As características desejáveis dos parâmetros cinéticos (alta Vmáx e baixa $\mathrm{K}_{\mathrm{M}} \mathrm{e}$ Cmín) foram verificadas em relação à cultivar Bico Ganga, nas concentrações de 20 e $40 \mathrm{mg} \mathrm{L}^{-1}$ de N, em ambas as épocas estudadas. Por sua vez, a cultivar Agulha, nas concentrações de 60 e $80 \mathrm{mg} \mathrm{L}^{-1}$ de $\mathrm{N}$, apresentou maiores valores desses parâmetros cinéticos, mesmo com menor desenvolvimento do sistema radicular.

Os maiores valores de Vmáx associados aos valores baixos de Cmín apresentados pela cultivar Bico Ganga, tanto aos 25 quanto aos 50 dias de idade, quando cultivada em solução nutritiva com $20 \mathrm{mg} \mathrm{L}^{-1} \mathrm{de} \mathrm{N}$, sugerem maior capacidade de absor- ção de $\mathrm{N}$ em condições de menor disponibilidade desse nutriente (Tabela 2), podendo ser uma indicação da adaptabilidade dessa planta a ambientes com baixa fertilidade natural.

\section{CONCLUSÕES}

1. A cultivar Bico Ganga mostra maior adaptação às condições de estresse nutricional dos trópicos úmidos.

2. A maior adaptação da cultivar Bico Ganga ao estresse nutricional manifesta-se por meio de maior crescimento radicular e maior eficiência de absorção de amônio a partir de solução nutritiva com baixas concentrações de $\mathrm{N}$.

3. A cultivar Agulha mostra maior capacidade de absorção de amônio sob condições de alta concentração de $\mathrm{N}$ na solução nutritiva.

\section{REFERÊNCIAS}

ANGHINONI, I.; VOLKART, C.R.; FATTORE, N.; ERNANI, P.R. Morfologia de raízes e cinética da absorção de nutrientes em diversas espécies e cultivares de plantas. Revista Brasileira de Ciência Solo, Campinas, v.13, n.3, p.355-361, set./dez. 1989.

BARRACLOUGH, P.B. Nutrient storage pool concentrations in plants as diagnostic indicators of nutrient sufficiency. Journal of Plant Nutrition, New York, v.16, n.3, p.195-198, 1993

CLAASSEN, N.; BARBER, S.A. A method for characterizing the relation between nutrient and concentration and flux into roots of intact plant. Plant Physiology, Rockville, v.54, n.4, p.564-568, Oct. 1974

CLARK, R.B. Effect of aluminum on growth and mineral elements of Al-tolerant and Al-intolerant corn. Plant and Soil, Dordrecht, v.47, n.3, p. 653-662, Aug. 1977.

CLARKSON, D.T. Factors affecting mineral nutrients acquisition by plants. Annual Review of Plant Physiology, Palo Alto, v.36, p.77-115, 1985. 
FERNANDES, M.S. Effects of environmental stress on the relationship of free amino-N to fresh weight of rice plants. Journal of Plant Nutrition, New York, v.14, n.11, p.1151-1154, 1991

FERNANDES, M.S. Effects of light and temperature on the nitrogen metabolism of tropical rice. Eas Lansing : Michingan State University, 1974. 80p Ph.D. Thesis.

FERRAZ JÚNIOR, A.S.L. Estudo do teor de proteína e eficiência de uso de $\mathbf{N}$ em cultivares de arroz (Oryza sativa L.). Itaguaí : UFRRJ, 1993. 186p. Dissertação de Mestrado.

FONTES, P.R.; BARBER, S.A. Crescimento do sistema radicular e cinética de absorção de fósforo pelo tomateiro, afetados por concentrações de fósforo na solução nutritiva. Pesquisa Agropecuária Brasileira, Brasília, v.19, n.10, p.1203-1210, out. 1984

FURLANI, A.M.C.; FURLANI, P.R. Composição e pH de soluções nutritivas para estudos fisiológicos e seleção de plantas em condições nutricionais adversas. Campinas : Instituto Agronômico, 1988. (IAC. Boletim Técnico, 121).

HOAGLAND, D.R.; ARNON, D.I. The water-culture method for growing plants without soil. Berkeley : University of California, 1950. p.1-32. (California Agricultural Experiment Station Circular, 347)

HOFFAND, E.; BLOEMHOF, H.S; LEFFELAAR, P.A.; FINDENEGG, G.R.; NELEMANS, S.A. Simulation of nutrient uptake by a growing root system considering increasing root density and inter-root competition. Plant and Soil, Dordrecht, v.124, n.2, p.149-155, June 1990.

JONES JUNIOR, J.B. Kjeldahl method for nitrogen (N) determination. Madison : Micro-Macro, 1991. p.21-66

LEE, R.B.; RUDGE, R.A. Effects of nitrogen deficiency on the absorption of nitrate and ammonium by barley plants. Annals of Botany, London, v.57, p.471-486, 1986.

MENDONÇA, M.L.S. Estudos dos mecanismos de tolerância ao alumínio e sua variabilidade genotípica em arroz (Oryza sativa L.). Itaguaí : UFRRJ, 1991. 176p. Dissertação de Mestrado.
MENGEL, D.B.; BARBER, S.A. Rate of nutrient uptake per unit of root under field conditions. Agronomy Journal, Madison, v.66, n.3, p.399-402, May/June 1974.

ROSSIELLO, R.O.P.; ARÚJO, A.P.; MANZATTO, C.V.; FERNANDES, M.S. Comparação dos métodos fotoelétricos e da interseção na determinação de área, comprimento e raio médio radicular. Pesquisa Agropecuária Brasileira, Brasília, v.30, n.5, p.633638, maio 1995

RUIZ, H.A. Estimativa dos parâmetros cinéticos em $\mathrm{K}_{\mathrm{M}} \mathrm{e}$ Vmáx por uma aproximação gráfico-matemática. Revista Ceres, Viçosa, v.32, n.179, p.79-84, jan. fev. 1985

RUIZ, H.A.; FERNANDES FILHO, E.I. Cinética: software para estimar as constantes Vmáx e $\mathrm{K}_{\mathrm{M}} \mathrm{da}$ equação de Michaelis-Menten. In: REUNIÃO BRASILEIRA DE FERTILIDADE DO SOLO E NUTRIÇÃO DE PLANTAS, 20., 1992, Piracicaba. Anais de simpósios. Campinas : Fundação Cargill, 1992. p.124-125. (Série Técnico-Científica, 180).

SATTELMACHER, B.; KLOTZ, F; MARSSCHNER, $\mathrm{H}$. Influence of nitrogen level on root growth and morphology of two potato varieties differing in nitrogen acquisition. Plant and Soil, Dordrecht, v.23, n.2 , p.131-137, Apr. 1990

SCHENK, M.K.; BARBER, S.A. Root characteristics of corn genotypes as related to $\mathrm{P}$ uptake. Agronomy Journal, Madison, v.71, n.6, p.921-924, Nov./Dec 1979

STEINGROBE, B.; SCHENK, M.K. Simulation of the maximum nitrate inflow (Imax) of lettuce (Lactuca sativa $\mathrm{L}$.) grown under fluctuating climatic conditions in the greenhouse. In: BARROW, N.J. (Ed.). Plant nutrition from genetic engineering to field practice. Dordrecht : Kluwer Academic, 1993 p. 177180.

TACHIBANA, J.; OHTA, Y. Root surface area as a parameter in relation to water and nutrient uptake by cucumber plant. Soil Science and Plant Nutrition, Tokyo, v.29, n.3, p.387-392, 1983.

VILELA, L.; ANGHINONI, I. Morfologia do sistema radicular e cinética de absorção de fósforo em cultivares de soja afetados pela interação alumínio-fósforo. Revista Brasileira de Ciência do Solo, Campinas, v.8, n.1, p.91-96, jan./abr. 1984 